\title{
Light ions in accelerator complex U70 of IHEP
}

\author{
Sergey Ivanov ${ }^{1,2}$ \\ Institute for High Energy Physics (IHEP) \\ 1, Nauki Sq., Protvino, Moscow Region, 142281, Russia \\ E-mail: Sergey.Ivanov@ihep.ru
}

The paper reports on the recent progress in implementing the program of accelerating light ions in the Accelerator Complex U70 of IHEP-Protvino. The list of milestones achieved since 2010 includes: (1) Proof-of-principle acceleration of carbon-12 to the top available $34.1 \mathrm{GeV} / \mathrm{u}$ (specific kinetic energy). (2) Circulation and slow extraction from the U70 of the carbon beam at flat-bottom $453-455 \mathrm{MeV} / \mathrm{u}$. (3) The first ever successful extraction of carbon nuclei at $24.1 \mathrm{GeV} / \mathrm{u}$ to the existing beam transfer line \#22 followed by feeding the FODS experimental facility (FOcusing Double-arm Spectrometer) with carbon beam for the start-up observations of relativistic nuclear physics events.

International Baldin Seminar on High Energy Physics Problems

September 10-15, 2012

JINR, Dubna, Russia

\footnotetext{
${ }^{1}$ Speaker

${ }^{2}$ On behalf of the IHEP light-ion task team
} 


\section{Introduction}

The program to accelerate light ions (deuterons, carbon nuclei) with a charge-to-mass ratio $q / A=0.4-0.5$ in the Accelerator Complex U70 of IHEP-Protvino, see Figs. 1, 2, aims at diversification and development of the accelerator facilities. The ion mode of operation involves a sequence of Alvarez DTL I100 ( 2 tanks of $3,4 \pi$ mode), rapid cycled synchrotron U1.5 $(162 / 3 \mathrm{~Hz}$ ), and the main synchrotron U70 itself (orbit length $1483.7 \mathrm{~m}$ ).

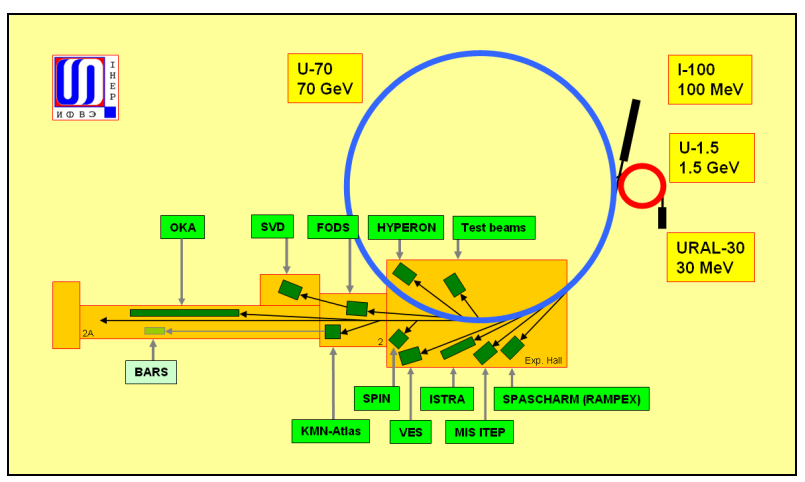

Figure 1: Accelerator Complex U70, beam transfer line network and fixed-target experimental facilities included. Proton mode (default) - cascade of URAL30-U1.5-U70, light-ion mode - I100-U1.5-U70.

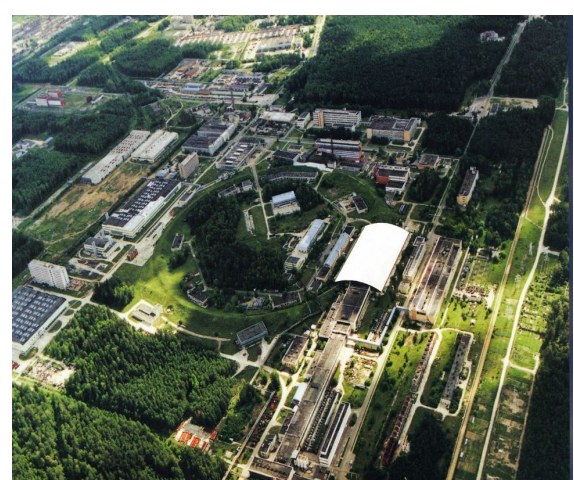

Figure 2: Aerial view of the Accelerator Complex U70 of IHEP.

This program is fulfilled incrementally, each recent machine run constituting a noticeable step in accomplishing the task.

This report overviews chronologically the progress achieved since 2010.

The starting point for this review is acceleration in the $\mathbf{U 7 0}$ of deuterons to the specific kinetic energy $23.6 \mathrm{GeV} / \mathrm{u}$ (flattop $8441 \mathrm{Gs)}$ ) with $5 \cdot 10^{10} \mathrm{dpp}$ obtained in Apr 27, 2010 during Run 2010-1. It was an outcome of sustained efforts re-launched since connecting the formerly stand-alone linac I100 to the U1.5 ring in Oct 2007.

Shortly in the aftermath of Run 2010-1, the cascade of I100, U1.5, and U70 involved was switched to the carbon-beam mode. The procedure implies re-assembly of the solid-state laser $\left(\mathrm{CO}_{2}, 5 \mathrm{~J}\right)$ ion source, acceleration of ions ${ }^{12} \mathrm{C}^{5+}$ in the $\mathrm{I} 100$ to $16.7 \mathrm{MeV} / \mathrm{u}$, thin-foil (Mylar, $4 \mu \mathrm{m}$ ) stripping to bare ions (nuclei) ${ }^{12} \mathrm{C}^{6+}$, and their subsequent acceleration in the synchrotrons $\mathrm{U} 1.5(6.9 \mathrm{~T} \cdot \mathrm{m}, 455 \mathrm{MeV} / \mathrm{u})$ and $\mathrm{U} 70(233 \mathrm{~T} \cdot \mathrm{m}, 34.1 \mathrm{MeV} / \mathrm{u})$.

\section{Run 2010-2}

During this run, on Dec 8, 2010, the fully stripped carbon ions ${ }^{12} \mathrm{C}^{6+}$ were first accelerated to $455.4 \mathrm{MeV} / \mathrm{u}$ (kinetic) in the U1.5. Beam intensity varied between 5.33.5.109 ipp through $26 \mathrm{~ms}$ ramp (once in $8 \mathrm{~s}$ ), Fig. 3.

There were, at least, two prerequisites for this success:

1. Operational experience gained earlier with the more intensive deuteron beam.

2. Abundance of ions delivered by the I100, Fig. 4. 


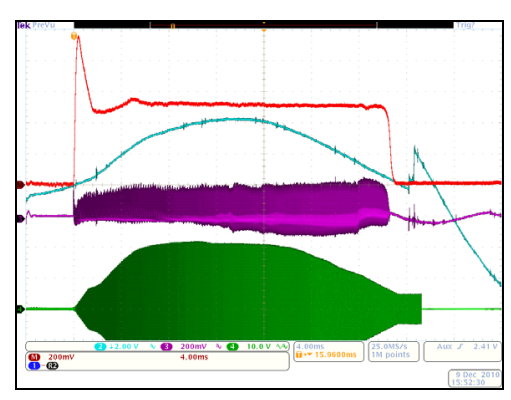

Figure 3: Carbon $\left({ }^{12} \mathrm{C}^{6+}\right)$ beam intensity monitored with a DCCT (upper (red) trace) and ramping rate of the U1.5 guide field (lower (blue) trace). In-out transfer is $65 \%$.

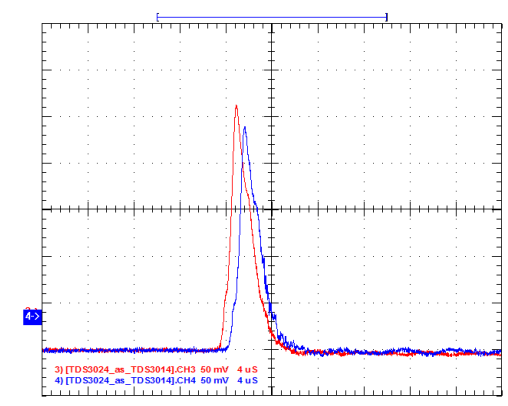

Figure 4: Beam at exits from the I100 (red) and BTL I100-U1.5 (blue). Pulsed current $21 \mathrm{~mA}$, max. Pulse length $5 \mu \mathrm{s}$. In-out transfer through the BTL is $90 \%$.

The first turns of carbon beam in the U70 at flat-bottom 353.1 Gs were committed on Dec 10, 2010, Fig. 5. Bunch length is $80 \mathrm{~ns} \mathrm{FW}$ at base. The lattice behaves as a magnetic ion separator, and $149.70 \mathrm{kHz}$ beam rotation frequency is a signature of the particular ${ }^{12} \mathrm{C}^{6+}$ ion species due to mass defect in a bound system of nucleons (Table 1).
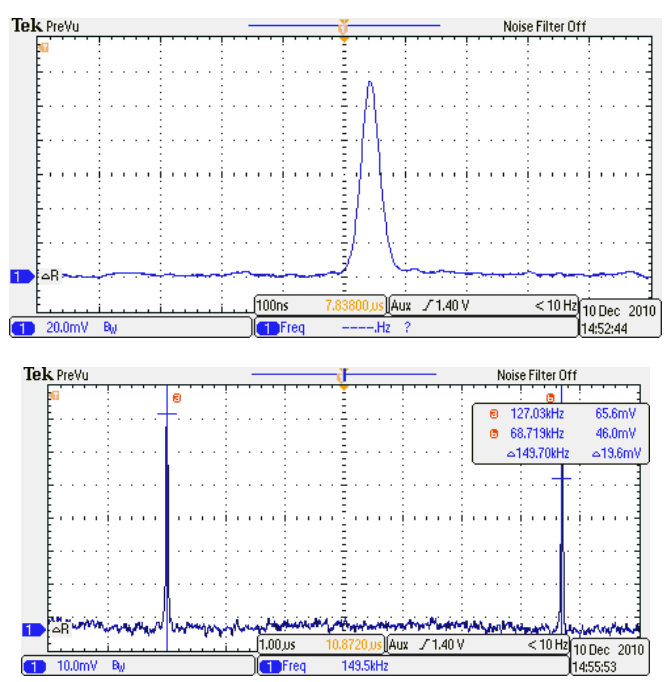
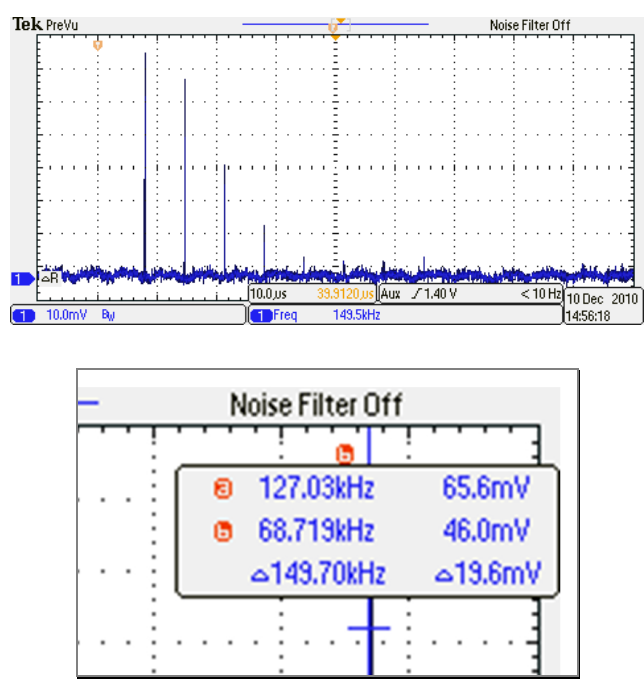

Figure 5: The first turns of carbon beam in the U70.

Table 1: Rotation frequency at 353.1 Gs flat-bottom in the U70

\begin{tabular}{|ll|}
\hline Ion species & Rotation frequency, $\mathrm{kHz}$ \\
\hline Carbon ${ }^{12} \mathrm{C}^{6+}$ & 149.70 \\
Deuterons ${ }^{2} \mathrm{H}^{1+}$ & 149.24 \\
\hline Protons ${ }^{1} \mathrm{H}^{1+}(p)$ & 183.80 \\
\hline
\end{tabular}

\section{Run 2011-1}

During this run, on Apr 24, 2011, carbon beam (a single bunch) was first accelerated in the U70 to the ultimate available energy of $34.1 \mathrm{GeV} / \mathrm{u}$ (flattop $12 \mathrm{kGs}$ ) with max $5 \cdot 10^{9} \mathrm{ipp}$ $(8 \mathrm{~s})$, design figure being $3 \cdot 10^{9}$ ipp. 
Organizationally, the ion-mode MD was very challenging with the use of a low-intensity pilot proton beam and multiple switching lattice power supply from rotor machine generators (RMG) to a stand-alone DC power supply unit (PSU) (130 A, $20 \mathrm{~kW})$ and back, refer to Table 2.

Table 2: Flow chart of regimes in Apr 21-27, 2011

\begin{tabular}{|lllll|}
\hline Field, Gs & 8590 & 352 & 12000 & 352 \\
\hline Power suppply & RMG & DC PSU & RMG & DC PSU \\
\hline$p$-beam & & & & \\
\hline C-beam & & & & \\
\hline
\end{tabular}

The I100 linac yielded some 12-14 mA (occasionally, up to $17 \mathrm{~mA}$ ) of pulsed current.

A remotely controlled tool to shift out spent emitting spot on the graphite cylinder surface inside the laser ion source was set in operation to ease in-run maintenance. That time, spot sustainability was some 800 cycles, or just $>1 \frac{1}{2} \mathrm{hr}$, a new spot requiring about 2 min of pretraining.

The U1.5 machine run smoothly and effectively.

A single bunch of ions was accelerated once per $8 \mathrm{~s}$. Its parameters complied with the best performance data for a proton mode: injected bunch length at base $\pm 60 \mathrm{~ns}$, fractional momentum spread $\pm 2 \cdot 10^{-3}$. Lifetime at flat-bottom is about $40 \mathrm{~s}$. Evolution of bunch length through cycle $\pm 60 \mathrm{~ns}$ (flat-bottom), $\pm 10 \mathrm{~ns}$ (transition crossing at $7.9 \mathrm{GeV} / \mathrm{u}$ kinetic), and $\pm 25 \mathrm{~ns}$ (flattop).

Figure 6 shows transition crossing as a "mountain range" display.

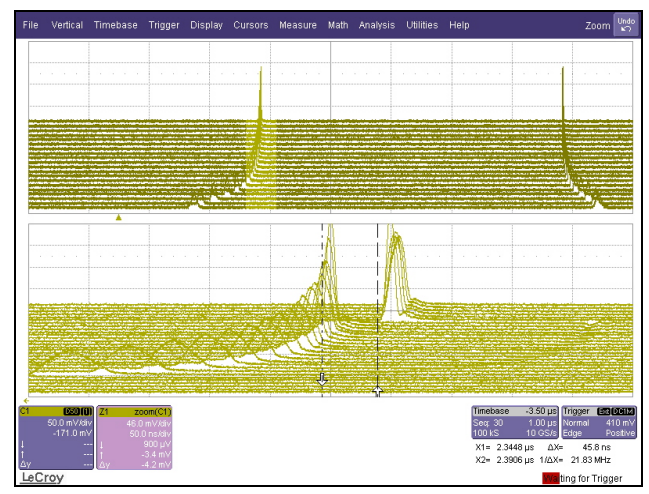

Scan period is 5000 turns. Scans are locked to the RF phase, and apparent jump of the bunch is, thus, virtual.

Figure 6: Transition crossing with $\mathrm{C}$ beam in the $\mathrm{U} 70$.

During end of the run 2011-1, the U70 operated in a storage-stretcher mode for a $453 \mathrm{MeV} / \mathrm{u}$ beam of ${ }^{12} \mathrm{C}^{6+}$ at $352 \mathrm{Gs}$ flat-bottom.

On Apr 25, 2011 this intermediate-energy beam was for the first time slowly extracted from the U70 ( $0.6 \mathrm{~s}$ long spills of $\left.1.5 \cdot 10^{9} \mathrm{ipp}\right)$. Both bunched (a single bunch) and un-bunched (azimuthally uniform) circulating beam options were tried.

Thus, the new slow extraction system was successfully beam-tested. This system is based on a compact cascade scheme "internal energy-degrader target IT\#28 — deflecting septum magnet SM\#34".

The beam is extracted inwards the U70 lattice from straight section SS\#34 pointing to the existing BTL\#6. This beam-line and its shielding are now dismounted. Instead, the new BTL\#25 is assembled there to deliver carbon beam for applied research (radiobiology, medicine, etc). 
Physically, the new extraction system at issue employs the well-known scheme by Piccioni-Wright, Ref. [1, 2], adapted for the strong-focusing synchrotron U70. In the latter case, a localized \& compact (only $1 / 20$ of orbit length) layout with $180^{\circ}$ betatron phase advances (both, horizontal and vertical) between IT and SM is feasible and suits the case.

It is the advantageous trade-off between ionization losses and hampering multiple Coulomb scattering (MCS) across the IT that opens a 'feasibility slot' for an effective (tens \% of in-out ratio) extraction of multi-charged ${ }^{12} \mathrm{C}^{6+}$ ions at flat-bottom of the U70, see Table 3. A test proton beam was also extracted with the scheme, naturally, with a lesser in-out ratio.

Table 3: Internal energy-degrader targets

\begin{tabular}{|lll|}
\hline Ion species & Carbon ${ }^{12} \mathrm{C}^{6+}$ & Protons $p$ \\
Kinetic energy, MeV/u & $353-355$ & 1320 \\
Target material & Beryllium & Graphite \\
Target thickness, mm & 4.0 & 30 \\
Target height, mm & 10 & 10 \\
Ionisation loss in momentum, $\Delta p / p_{0}$ & -0.0069 & -0.0069 \\
R.m.s. MCS scattering angle, $\mathrm{mrad}$ & 0.96 & 2.93 \\
\hline
\end{tabular}

The new flat-bottom extraction system was proposed, manufactured, installed and beamtested (proton and carbon ions) within a tight schedule starting in Jan 2010. Beam spot size observed at exit flange of SM\#34 well fits to the design figures - around $20 \times 10 \mathrm{~mm}^{2}(\mathrm{~h} \times \mathrm{v})$ for azimuthally uniform and $10 \times 10 \mathrm{~mm}^{2}$ for bunched beams.

In course of beam tests, two ways of pushing the waiting beam onto the IT\#28 for slow extraction were tested.

The first one applies varying (dynamical) localized closed-orbit distortions (bumps) near IT\#28 and SM\#34, Fig. 7.

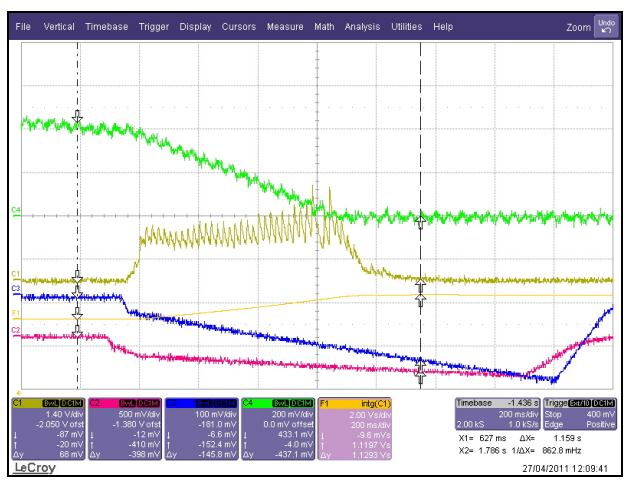

Traces from top to bottom:

(1) circulating $\mathrm{C}$ beam intensity; (2) slow spill (a saturated signal); $(3,4)$ coil currents for closed-orbit bumps near IT\#28 and SM\#34, resp.

Figure 7: Slow spill under bumps of closed orbit.

The second way is to apply horizontal betatron noise causing controlled beam diffusion towards IT\#28. To this end, the existing electrostatic deflector ESD\#2, otherwise servicing the transverse feedback, is driven by a noise with flat (within $1.5-70 \mathrm{kHz}$ bandwidth) power spectrum, see Fig. 6 and Ref. [3]. This option looks more promising for future routine operation due to inherent stationarity of beam traces. 


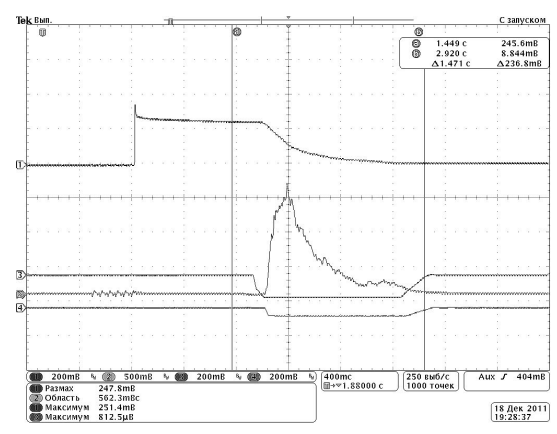

Traces from top to bottom:

(1) circulating $\mathrm{C}$ beam intensity; (2) slow spill (a proportional signal); $(3,4)$ coil currents for steady closed-orbit bumps near IT\#28 and SM\#34, resp.

Figure 8: Stochastic slow spill.

\section{Run 2011-2}

During this ion-beam run, the U70 operated in a storage-stretcher mode for a $455 \mathrm{MeV} / \mathrm{u}$ $\mathrm{C}$ beam at $353 \mathrm{Gs}$ flat-bottom. Top beam intensity gained was $10 \cdot 10^{9} \mathrm{ipp}$, well exceeding the design figure of $3 \cdot 10^{9}$ ipp. Efforts were spent to get more experience with the new slow extraction, the first observations of Bragg's range in a substance being recorded.

Two experimental shots are shown in Figs. 9, 10. Figure 11 confirms the expected performance of the slow extraction.

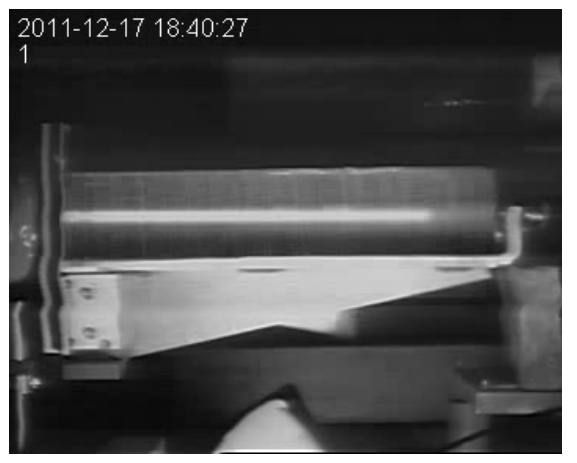

Figure 9: Finite range $(30 \mathrm{~cm} \mathrm{ca})$ of extracted carbon beam through a plastic scintillator at $455 \mathrm{MeV} / \mathrm{u}$.

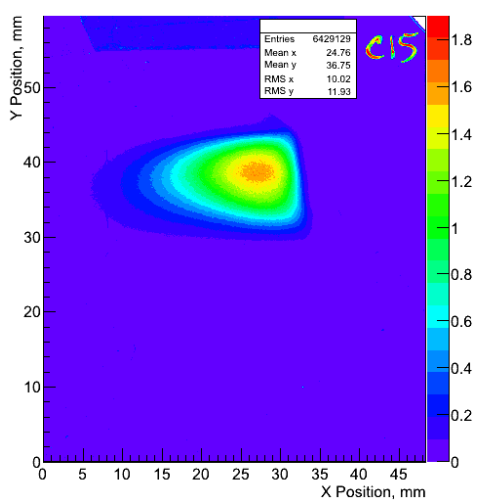

Figure 10: Bunched $\mathrm{C}$ beam spot at exit from SM\#34 under stochastic slow extraction. Convolution over 3 cycles $8 \mathrm{~s}$ long each.

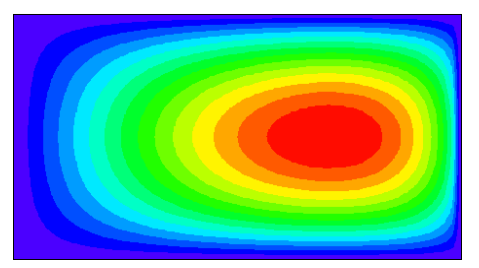

Surface contour plot of

$$
\begin{aligned}
& F(x, y)=C \cdot x \cdot \sqrt{1-x^{2}} \times \\
& \quad \times\left(1-y^{2}\right),|x, y| \leq 1
\end{aligned}
$$

Figure 11: Calculated beam spot profile for a parabolic bunched beam under stochastic slow extraction. 


\section{Run 2012-1}

During the latest run 2011-2, carbon beam was accelerated to $24.1 \mathrm{GeV} / \mathrm{u}$ (flattop $8590 \mathrm{Gs})$ with $5 \cdot 10^{9}$ ipp ( $\left.8 \mathrm{~s}\right)$.

The I100 team has managed to improve drastically the performance of the laser ion source. Thorough choice of gas mixture composition and laser pulse energy now allows to operate with 10-12 mA of pulsed current for 4000 cycles per an emitting point (instead of the former 800 cycles), i.e. for $>8 \mathrm{hr}$.

All the high-energy beam extraction systems available in the U70 - fast single-turn, slow resonant (both, via lens Q38 and stochastic), slow with a bent Si-crystal deflector - were readily tested with a carbon beam.

The beam thus extracted was transferred through the existing $190 \mathrm{~m}$ long BTL\#22 to the FODS experimental facility (the FOcussing Double-arm Spectrometer). The primary beam and its fragments were detected with the FODS scintillator counters, calorimeter, monitor and data acquisition systems. The counters and calorimeter were pre-calibrated with a $49.1 \mathrm{GeV}$ (kinetic) pilot proton beam.

To start with, the BTL\#22 was tuned to $50 \mathrm{GeV} / \mathrm{c}$ central momentum (protons). In this case, it accepts $25 \mathrm{GeV} / \mathrm{c} / \mathrm{u}$ beam of ion species with charge-to-mass ratio $q / A=1 / 2$ as well $\left({ }^{2} \mathrm{H}^{1+}\right.$, $\left.{ }^{4} \mathrm{He}^{2+},{ }^{6} \mathrm{Li}^{3+},{ }^{10} \mathrm{~B}^{5+}\right)$. Indeed, the hadron calorimeter saw peaks in energy spectrum at $300 \mathrm{GeV}$ (12 nucleons $\times 25 \mathrm{GeV} / \mathrm{u}$ (full energy) in $\left.{ }^{12} \mathrm{C}\right), 100 \mathrm{GeV}\left({ }^{4} \mathrm{He}\right)$ and $50 \mathrm{GeV}\left({ }^{2} \mathrm{H}\right)$, refer to Fig. 12 (left).

Then, to detect fragments with $q / A<1 / 2$, the BTL\#22 was re-tuned to a higher momentum $60 \mathrm{GeV} / \mathrm{c}$ (protons) and fractional momentum acceptance $\pm 1 \%$. In this case, of all ${ }^{12} \mathrm{C}$ fragments, it can accommodate and transfer ${ }^{7} \mathrm{Li}$ nuclei only. Those indeed show themselves up as $175 \mathrm{GeV}$ peak in the calorimeter readouts, Fig. 12 (right).

Fragmentation of ${ }^{12} \mathrm{C}$ occurs, presumably, due to an unattended presence of substances across the beam path (foils, air gaps, beam monitors, septa, etc).

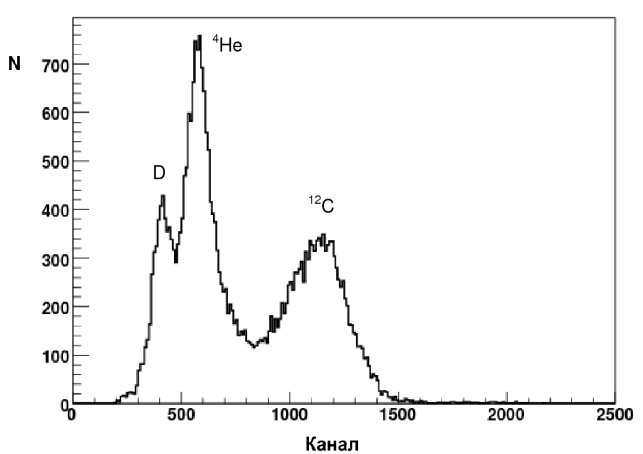

viaBTL\#22@50GeV/c (p)

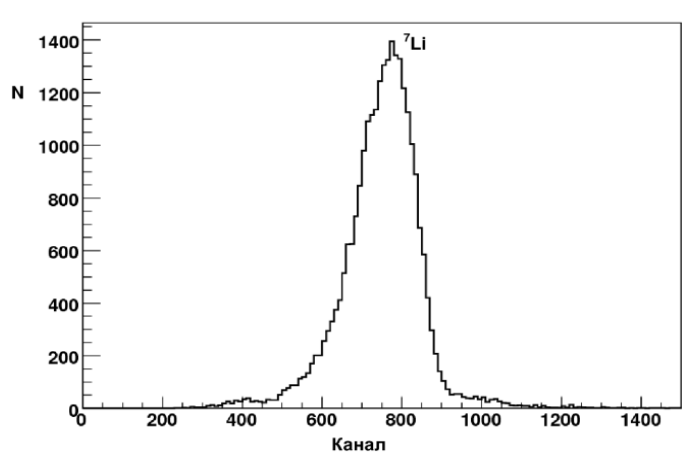

via BTL\#22@60 GeV/c $(p)$

Figure 12: Amplitude spectra from the FODS hadron calorimeter. Fragmentation of the carbon nuclei. Abscissa division value is 3.2 bins per a $\mathrm{GeV}$.

Thus, operational retuning of optics (central momentum and acceptance) of the BTL\#22 allowed to use this beam-line as an 'ad hoc' Fragment Separator yielding the first ever experimental observations of high-energy nuclear-physics events obtained with a $300 \mathrm{GeV}$ (full energy) carbon beam delivered by the U70 (Apr 27, 2012). 


\section{Conclusion}

In course of the four recent machine runs (2010-12) reported, important milestones of the program to accelerate beams of bare carbon ions in the Accelerator Complex U70 of IHEPProtvino were achieved. Every run had its specific highlight boldfaced in the text above.

The light-ion (carbon) program pursues two goals:

1. To accelerate, extract and deliver high-energy (24.1-34.1 GeV/u, kinetic) carbon beam for fixed-target experiments in relativistic nuclear physics.

2. To accelerate, extract and deliver intermediate-energy (453-455 MeV/u and less) carbon beam for experimental applied research, including radiobiology and radiation medicine.

Steps are taken in either direction. Still, some advances have a flavor of a feasibility 'proof-of-principle' experiment yet. More efforts are planned to elaborate the techniques and attain a reliable routine operation with the better light-ion beam performance data.

\section{Acknowledgement}

Advances of the light-ion program in the Accelerator Complex U70 of IHEP are the results of sustained efforts of the dedicated task team comprising:

N. Tyurin, A. Zaitsev, A. Soldatov, A. Afonin, Yu. Antipov， G. Britvich，A. Bulychev, A. Ermolaev, V. Garkusha, G. Hitev, N. Ignashin, D. Khmaruk, V. Kryshkin, V. Lapygin, O. Lebedev, V. Ledenev, E. Ludmirsky, A. Maximov, Yu. Milichenko, A. Minchenko, V. Seleznev, V. Stolpovsky, I. Sulygin, S. Sytov, G. Kuznetsov, and others.

This report is presented on behalf of them all.

\section{References}

[1] O. Piccioni, D. Clark, R. Cool, G. Friedlander, and D. Kassner, External Proton Beam of the Cosmotron. Rev. Sci. Instruments. 26, 232 (1955).

[2] B.T. Wright, Magnetic Deflector for the Bevatron, Rev. Sci. Instruments, 25, 429 (1954).

[3] S. Ivanov, O. Lebedev, Transverse Noise Blow-Up of Beam in the U-70 Synchrotro,, Preprint IHEP 2012-10, Protvino, 2012. 